\title{
Etude de l'interaction fluide-structure en érosion de cavitation
}

\author{
par J.L. Reboud et R. Fortes Patella
}

Laboratoire des Ecoulements Géophysiques et Industriels, Grenoble, France

\section{I —INTRODUCTION}

La difficulté d'analyse du phénomène d'érosion provoqué par la cavitation est liée pour d'une grande partie à la diversité des formes d'agression qui peuvent affecter le matériau. La complexité des phénomènes qui se produisent au moment de l'implosion de structures de vapeur au voisinage des parois solides, à des échelles extrêmement réduites tant en temps qu'en espace, limitent encore les possibilités d'investigation expérimentale directe. L'implosion d'une simple bulle à proximité d'une paroi est en effet accompagnée par la formation d'une onde de choc et/ou d'un jet rentrant dirigé vers la paroi, de rebonds et d'implosions successifs accompagnés du fractionnement en bulles multiples. L'absence d'observation directe des derniers instants de tels processus alimente encore actuellement l'incertitude quant au scénario exact conduisant à la formation d'une ou plusieurs empreintes dans un matériau soumis à l'implosion d'une bulle isolée, et a fortiori au processus complet d'érosion par un écoulement cavitant.

L'analyse d'échantillons soumis à l'agression d'une structure de cavitation pendant une durée suffisamment courte pour n'occasionner qu'un nombre identifiable d'empreintes est considérée depuis longtemps [1] comme un moyen de caractériser de façon globale le potentiel érosif d'un écoulement. Elle a été utilisée en particulier pour proposer des lois de similitudes globales permettant de relier les caractéristiques d'un écoulement cavitant, les propriétés physiques d'un matériau et le taux de marquage de la surface exposée [2], [3], [4]. En parallèle à cette démarche globale, l'utilisation de relevés métrologiques de la forme des empreintes pour tenter de déterminer la nature de l'impact ayant conduit à leur formation est étudiée au sein du laboratoire depuis plusieurs années [5], [6]. Pour ce faire, le développement de modèles de simulation d'impacts hydrodynamiques liés à l'implosion de structures de vapeur et de déformation plastique d'une surface solide soumise à de tels impacts a été entrepris. La comparaison entre la déformation du solide modélisée numériquement et celle observée expérimentalement sur plusieurs matériaux soumis à la cavitation a été réalisée. Elle a permis d'identifier l'onde de surpression sphérique comme auteur le plus probable des déformations relevées et de déterminer les caractéristiques hydrodynamiques du mécanisme responsable des endommagements observés [7].

L'étude de l'interaction fluide-structure en érosion de cavitation est réalisée au moyen du couplage d'un modèle numérique de réponse dynamique d'un solide élastoplastique soumis à des chargements transitoires rapides (code SOLIDE [5]), et d'un code de calcul en fluide compressible, développé pour étudier les phénomènes de propagation dans un liquide d'ondes de surpression de grande amplitude (code FLUIDE [6]).

\section{II — MODÉLISATION NUMÉRIQUE}

Le code de calcul SOLIDE mis en œuvre, présenté en détail dans [5] et [7], est issu d'un modèle théorique et numérique du comportement dynamique d'un matériau, traité comme un milieu continu, dont la loi de comportement décrit le phénomène d'hystérésis élastoplastique [8]. Pour les petites déformations, cette loi est tangente au comportement élastique faisant intervenir le module d'Young $E$ et le coefficient de Poisson v. Pour les fortes sollicitations elle tend vers le comportement plastique décrit par le critère de Von Mises et la contrainte limite de plasticité So. Le code fournit l'évolution transitoire et l'état final des champs des contraintes, des déformations et des différentes formes d'énergie dans le matériau, sous l'hypothèse de symétrie axiale. Il ne prend en compte ni les phénomènes de rupture ni ceux de perte de masse et ne peut donc simuler que la période d'incubation de l'érosion de cavitation.

Différents types de chargements transitoires rapides peuvent être appliqués à la surface du matériau comme condition limite sur la face supérieure du domaine de calcul. Une condition de symétrie est appliquée à l'axe. Pour les deux frontières restantes, une condition de sortie libre des ondes se propageant au sein du matériau a été développée de façon à éviter les réflexions parasites. Lors d'un impact à la frontière supérieure du matériau, une partie de l'énergie fournie au solide est évacuée par les ondes élastiques. Lorsque l'amplitude est suffisante pour atteindre un niveau de plastification significatif, des déformations et des contraintes rési- 


\section{NOMENCLATURE}

$\varepsilon$

$\rho, \rho_{s}$

$\sigma$

$E c_{-} l, E c_{-}$

Eext_l

Eent_s

Eint_s

$h$

$P, P \max$

$R 10 \%$

$V_{1}, V_{s}$

Ur, Uz tenseur des déformations dans le solide

masses volumiques du liquide et du solide

tenseur des contraintes dans le solide

énergie cinétique dans le liquide (volume $V_{1}$ ) et dans le solide (volume $V_{s}$ )

énergie sortant du volume liquide par les trois frontières

énergie entrant dans le volume solide par l'interface liquide-solide

énergie des efforts intérieurs dans le solide (volume $V_{s}$ )

profondeur maximale d'une empreinte

pression dans le liquide, pression maximale à la paroi du solide

rayon d'une empreinte à $10 \%$ de sa profondeur maximale $h$

volumes des domaines liquide et solide

composantes radiale et verticale de la vitesse dans le liquide
[]

$\left[\mathrm{kg} / \mathrm{m}^{3}\right]$

$[\mathrm{Pa}]$

[J]

[J]

$[\mathrm{J}]$

[J]

$[\mathrm{m}]$

$[\mathrm{Pa}]$

[m]

$\left[\mathrm{m}^{3}\right]$

$[\mathrm{m} / \mathrm{s}]$ duelles subsistent dans la région de l'impact, associées à la part restante de l'énergie fournie au matériau et à une déformation permanente de la surface solide [9]).

Pour la modélisation de l'émission et de la propagation d'ondes de surpression sphériques dans l'eau, un code de calcul FLUIDE a été mis en œuvre [6]. Il traite les équations d'Euler instationnaires en coordonnées cylindriques, pour un fluide compressible dont le comportement répond à une équation d'état empirique barotrope du type :

$$
\frac{P+B}{P_{\infty}+B}=\left(\frac{\rho}{\rho_{\infty}}\right)^{n}
$$

où $B=3.10^{8} \mathrm{~Pa}$ et $n=7,15$ pour l'eau. Dans ce modèle simple appliqué à l'eau froide l'équation de l'énergie n'est pas traitée, ce qui suppose que les effets thermiques restent négligeables. Ce point pourra faire l'objet d'améliorations ultérieures. Dans le cas d'un maillage rectangulaire régulier, la discrétisation utilisée est de second ordre en espace (schéma de Van Leer [10]) et la résolution en temps fait appel à un schéma explicite. Les conditions aux limites de ce problème hyperbolique sont données par l'approche de Thomson [11]. Elles permettent en particulier d'autoriser la sortie libre des ondes de pression hors du domaine de calcul, ou d'imposer à l'interface avec le solide la composante normale de la vitesse.

Pour modéliser les effets de l'interaction entre le fluide et le solide, les codes de calcul décrits ci-dessus ont été cou-

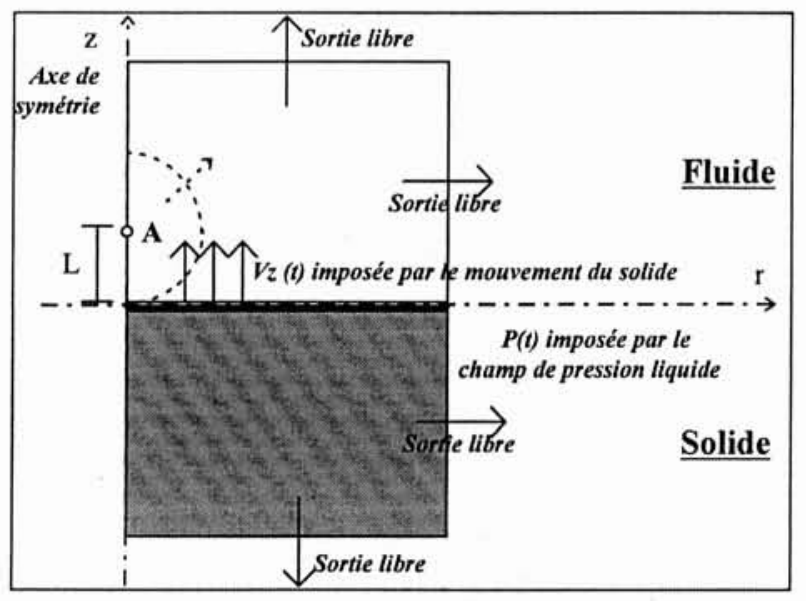

1. Schéma des domaines de calculs, couplage fluidesolide (coordonnées cylindriques, axe à gauche). plés. Les deux codes adoptent des formulations explicites pour le calcul de l'évolution temporelle, si bien qu'une formulation du même type peut être employée pour leur couplage. Les calculs sont effectués à chaque pas de temps dans le fluide et dans le solide. Les conditions de pression à la paroi, résultant du calcul FLUIDE à chaque itération, sont imposées comme condition à la paroi supérieure du solide. A l'opposé, les vitesses de recul de la paroi données par le calcul SOLIDE sont utilisées en conditions limites sur la frontière correspondante du domaine fluide. Si les vitesses de recul peuvent atteindre des valeurs considérables, la durée extrêmement brève du phénomène rend le déplacement de la paroi solide assez peu important. Du fait de la formulation lagrangienne du modèle SOLIDE, les déformations sont naturellement prises en compte. Pour le code FLUIDE, par contre, une approximation a été faite en négligeant ce déplacement et en appliquant les conditions aux limites aux næuds du maillage fixe. Ainsi, le recul de la paroi se traduit par un débit liquide sortant du domaine de calcul et les deux domaines maillés ne sont plus exactement en contact.

\section{IINTERACTION ENTRE UNE ONDE DE SURPRESSION ET UN SOLIDE ÉLASTOPLASTIQUE}

A titre d'exemple, on a choisi de présenter ici la simulation de la génération d'une empreinte de petite taille (de l'ordre de $2 \mu \mathrm{m}$ de profondeur et un dixième de millimètre de rayon) générée dans de l'aluminium pur, matériau assez peu résistant (faibles valeurs pour: la masse volumique $\rho=2700 \mathrm{~kg} / \mathrm{m}^{3}$; le module d'Young $E=50 \mathrm{GPa}$ et la contrainte limite : $S o=100 \mathrm{MPa}$ ).

\subsection{Caractéristiques de l'onde de surpression}

L'onde de surpression émise dans l'eau a été simulée numériquement sur un maillage régulier, pour assurer une résolution au second ordre et réduire ainsi au minimum la dissipation numérique parasite. Il comporte 61 x 61 mailles de $10 \mu \mathrm{m}$ de côté. La pression initiale est uniforme et vaut $P_{0}=0,1 \mathrm{MPa}$. Par analogie avec le phénomène d'implosion d'une bulle, l'onde de pression est générée en initialisant un champ de vitesse convergent vers un point du domaine liquide (A sur le schéma de la figure 1) dont la distance à la paroi solide est notée $L$. La compressibilité du fluide se charge au cours des itérations suivantes de générer une surpression dans cette zone, qui se propage alors sous forme d'une onde sphérique. Le champ de vitesses convergentes 
initial est fixé de façon à assurer la conservation du débi entre les différentes surfaces sphériques concentriques et à vérifier une condition d'imperméabilité en présence d'une paroi. Une valeur de la vitesse initiale doit être imposée en un point du domaine, et peut être réglée pour modifier l'amplitude de l'onde de pression émise. A partir de l'instant initial du calcul, une surpression est générée autour du point de convergence initial, et se propage radialement au sein du liquide. Ses caractéristiques dépendent de la compressibilité du milieu réglée par la loi d'état (1).

Pour pouvoir suivre l'évolution de l'onde en milieu infini, une première simulation a été réalisée sans prendre en compte la paroi solide. La pression émise se propage en décroissant avec le rayon. Compte tenu de l'amplitude élevée, les effets non linéaires se font sentir, qui se traduisent par un redressement du signal, une célérité supérieure à celle du son et une décroissance moyenne de l'amplitude avec le rayon de l'ordre de $r^{-1,5}$ pour une amplitude de l'ordre de 0,1 à $1 \mathrm{GPa}$ et même $r^{-2}$ pour les pressions supérieures au Gigapascal.

Le temps de passage de l'onde émise est de l'ordre de 30 $\mathrm{ns}$, valeur croissante avec le rayon pendant les premiers instants de la propagation, à cause de la décroissance de l'amplitude de 1 'onde plus rapide que $1 / r$. Ce phénomène s'atténue au fur et à mesure de la diminution de l'amplitude du signal. Bien que la simulation soit réalisée en ne traitant qu'une seule phase, les valeurs obtenues sont d'un ordre de grandeur conforme à celles rapportées par Vogel et al. [12] ou Ward et Emmony [13] lors de leurs mesures de transitoires acoustiques liés à l'implosion de bulles de vapeur.

\subsection{Interaction onde-paroi}

En présence de la paroi solide, on vérifie après dépouillement de plusieurs simulations que la pression maximale $P \max$ appliquée sur la surface est croissante avec la vitesse concentrique initiale. Pour la valeur de $L$ de $0,07 \mathrm{~mm}$, un pic de pression de l'ordre de $1 \mathrm{GPa}$ à la paroi solide est obtenu pour une vitesse convergente initiale de l'ordre de $1000 \mathrm{~m} / \mathrm{s}$ à la distance $L / 2$ du point central. Une telle pression crée une empreinte d'environ $2 \mu \mathrm{m}$ de profondeur. La valeur de vitesse obtenue pour ce choix délibéré de génération d'une petite empreinte dans un matériau peu résistant laisse entrevoir les difficultés auxquelles on peut s'attendre pour décrire le comportement physique du fluide dans les conditions extrêmes nécessaires à la génération de trous importants dans un matériau à forte contrainte limite de plasticité.

La figure 2 présente, en coordonnées cylindriques axisymétriques, le champ de pression dans le fluide (en haut) et les champs de contraintes dans le solide (en bas) à trois instants successifs. On observe l'émission de l'onde de surpression qui se propage dans l'eau et qui frappe la paroi solide ainsi que la réponse du matériau à l'impact de l'onde.

Des distributions instantanées de pression à la paroi sont tracées (fig. 3) à différents instants régulièrement espacés. Les forts gradients de pression générés le long de la paroi solide créent des vitesses radiales d'écoulement de plusieurs centaines de mètres par seconde. Une amélioration du modèle consisterait à prendre en compte la viscosité du fluide et le cisaillement ainsi créé à la surface du solide dans les deux modèles.

Dans le cas de l'aluminium, le recul de la paroi sous l'impact de l'ordre de $1 \mathrm{GPa}$ s'effectue au niveau de l'axe de symétrie avec une vitesse de l'ordre de $100 \mathrm{~m} / \mathrm{s}$, pendant le passage de l'onde. Une telle valeur entraîne un affaiblissement de l'amplitude du pic de pression ressenti par la paroi. A titre de comparaison, un calcul effectué sans couplage (paroi considérée comme rigide pour le calcul FLUIDE) conduit à un niveau de surpression de 15 à $20 \%$ supérieur à celui prenant en compte le mouvement du solide et à une profondeur d'empreinte plus importante (fig. 4). Il est clair que ce rapport augmente lorsque l'amplitude de pression est plus grande. Pour les matériaux plus denses et plus durs que l'aluminium, ce pourcentage doit bien entendu être considéré comme un majorant. La profondeur finale obtenue est généralement légèrement inférieure à celle atteinte en cours d'impact. L'élasticité du milieu restitue ainsi une part de la déformation lors de la décharge de la surface.

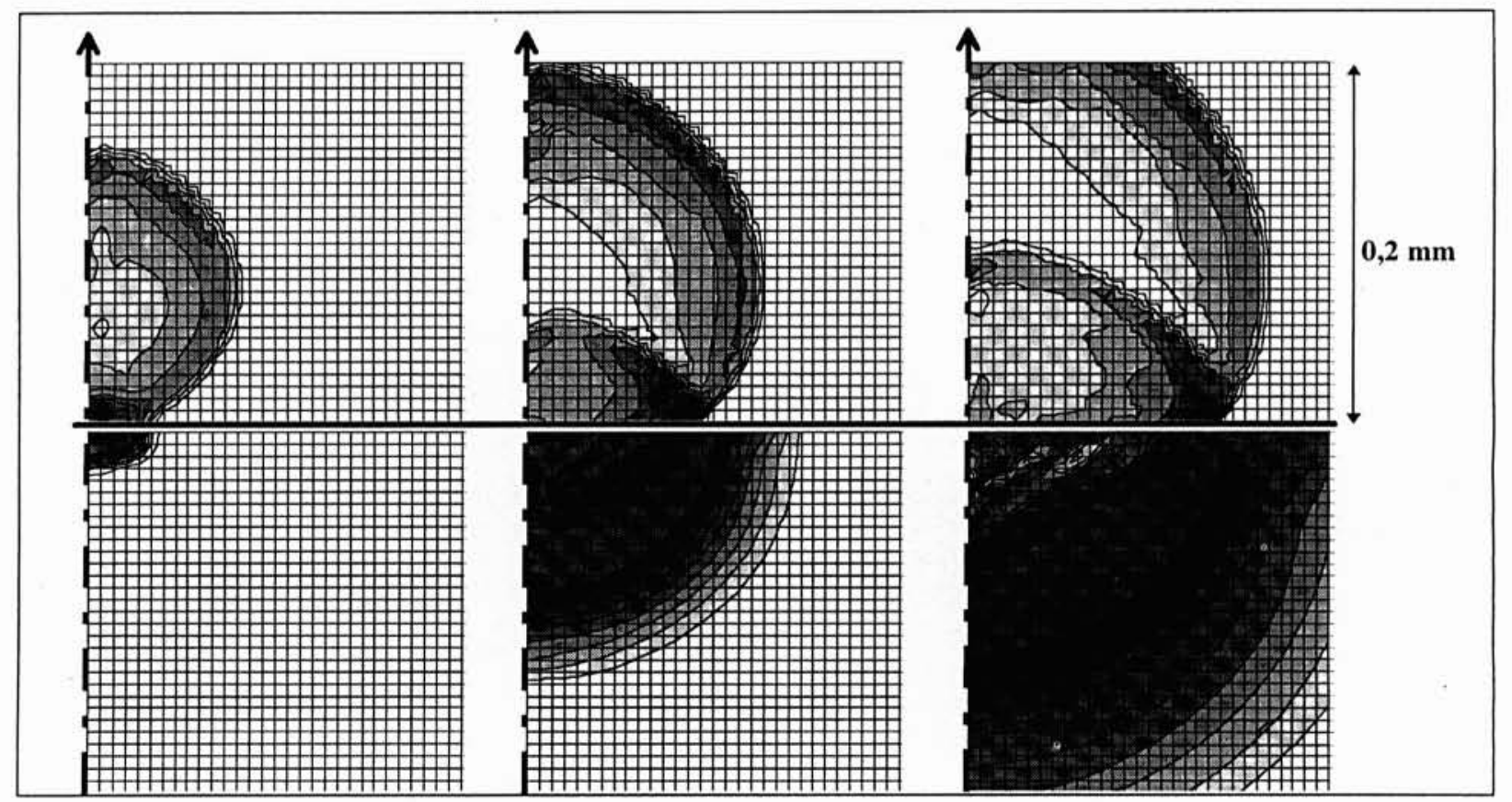

2. Couplage Fluide-Structure - illustration des champs de pression dans le liquide (en haut) et des champs de contraintes ( $2^{\mathrm{e}}$ invariant du déviateur de $\sigma$ ) dans le matériau (en bas) à trois instants successifs espacés de $24 \mathrm{~ns}$. 


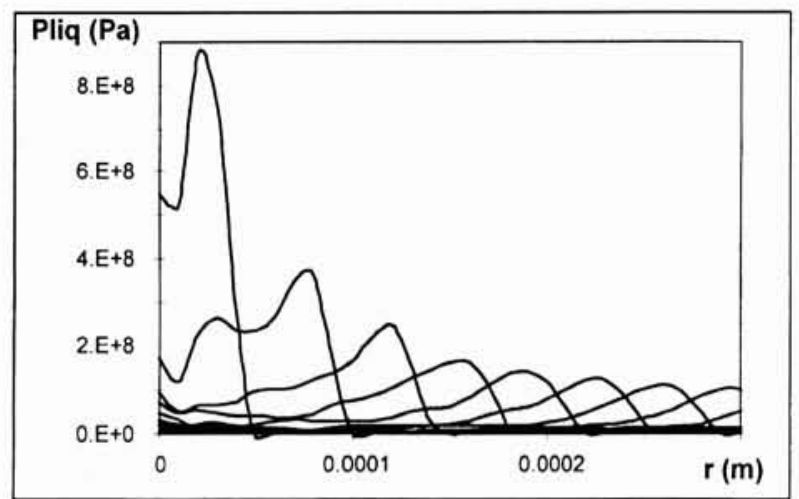

3. Distribution de pression le long de la paroi à des instants successifs espacés de $24 \mathrm{~ns}$ (les trois distributions de gauche correspondent aux illustrations de la figure 2).

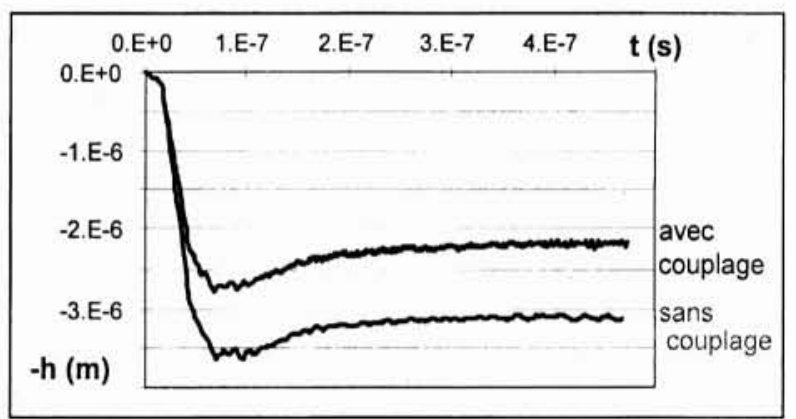

4. Evolution temporelle de la profondeur au centre de l'empreinte au cours de l'impact.

\section{- 3.3 Bilan d'énergie}

Pour réaliser un bilan énergétique de l'interaction, les différentes formes d'énergie sont intégrées sur les volumes liquide et solide $V_{1}$ et $V_{\mathrm{s}}$. L'énergie cinétique rend compte du mouvement des milieux et est utilisée en particulier pour constater la sortie des ondes du domaine de calcul aussi bien pour le liquide que pour le solide :

$$
E c \_s=\int_{V_{s}}^{\rho} \frac{s}{2}\left(U r^{2}+U z^{2}\right) d V ; E c \_l=\int_{V_{l}} \frac{\rho}{2}\left(U^{2}+U z^{2}\right) d V ;
$$

L'énergie des efforts intérieurs dans le solide est obtenue par intégration en temps de la puissance des efforts intérieurs, produit des tenseurs de contrainte et de vitesse de déformation :

$$
\text { Eint_s }=\int_{0}^{t}\left(\int_{V s} \operatorname{tr}(\sigma \varepsilon) d V\right) d t
$$

L'énergie interne dans le liquide dépend quant à elle des variations d'enthalpie $\Delta h$, déduites de la relation (1) sous conditions isentropiques et des variations du champ de pression $\Delta P=P-P_{\infty}$ :

$$
E i_{-} l=\int_{V l}\left(\Delta h-\frac{\Delta P}{\rho}\right) \rho d V, \operatorname{avec} \Delta h=\frac{n\left(P_{\infty}+B\right)}{(n-1)}\left[\left(\frac{P+B}{P_{\infty}+B}\right)^{(n-1) / n}-1\right.
$$

L'énergie totale dans le milieu liquide vaut alors :

$$
\text { Etot_l }=E i_{-} l+E c_{-} l
$$

Pour compléter le bilan, on s'intéressera à Eext_l qui représente la somme des flux d'enthalpie et d'énergie cinétique sortant du domaine de calcul fluide par les trois surfaces frontières (deux conditions de sortie libre et l'interface liquide-solide), ainsi qu'à l'énergie Eent_s entrant dans le solide par la surface de contact avec le milieu liquide.

La figure 5 illustre ces différentes formes d'énergie et leur évolution au cours du temps. Compte tenu des conditions initiales imposées, l'énergie cinétique dans le liquide $E c_{-} l$ est maximale en début de calcul, puis décroît rapidement lors de la génération de l'onde de surpression, pour tendre vers 0 à la fin du calcul lorsque l'onde sort du domaine maillé. L'énergie totale Etot_l à l'intérieur du domaine liquide et le flux à sa frontière Eext_l présentent des évolutions opposées, ce qui traduit la conservation de l'énergie initialement fournie (on note à ce propos sur Etot_l des oscillations dans les instants suivant l'initialisation, qui proviennent du transitoire initial particulièrement brutal qui n'est pas actuellement parfaitement traité par le code). L'évolution du flux Eext_l présente trois zones marquées, correspondant à l'arrivée de l'onde successivement à la surface du solide, à la frontière supérieure, puis à la frontière latérale du domaine de calcul. Dans la première partie de son évolution on note bien que le flux sortant du domaine fluide correspond à celui entrant dans le domaine solide Eent_s. Dans celui-ci on note que l'énergie cinétique $E c \_s$ reste assez faible et disparaît rapidement de la zone d'impact après la décharge. L'énergie des efforts intérieurs Eint_s croît rapidement, absorbant dans la phase de chargement la quasi-totalité du flux d'énergie Eent $s$ entrant dans le matériau. L'énergie des efforts intérieurs se stabilise ensuite, après une légère décroissance correspondant au rappel élastique. La valeur finale stabilisée représente l'énergie subsistant sous forme de déformations permanentes et des contraintes résiduelles associées à l'hétérogénéité du niveau de plasticité atteint dans le matériau. La différence finale entre Eent_s et Eint_s représente la quantité d'énergie dispersée dans le matériau sous forme d'ondes élastiques, longitudinales, transversales ou de surface, s'éloignant du point d'impact et éliminées du domaine de calcul solide par la condition de sortie libre. Dans le cas présenté, on note que le rapport entre l'énergie transmise au matériau Eent_s et l'énergie totale Etot $l$ fournie par l'onde de surpression dans le liquide est d'environ $30 \%$, ce qui correspond bien au fait

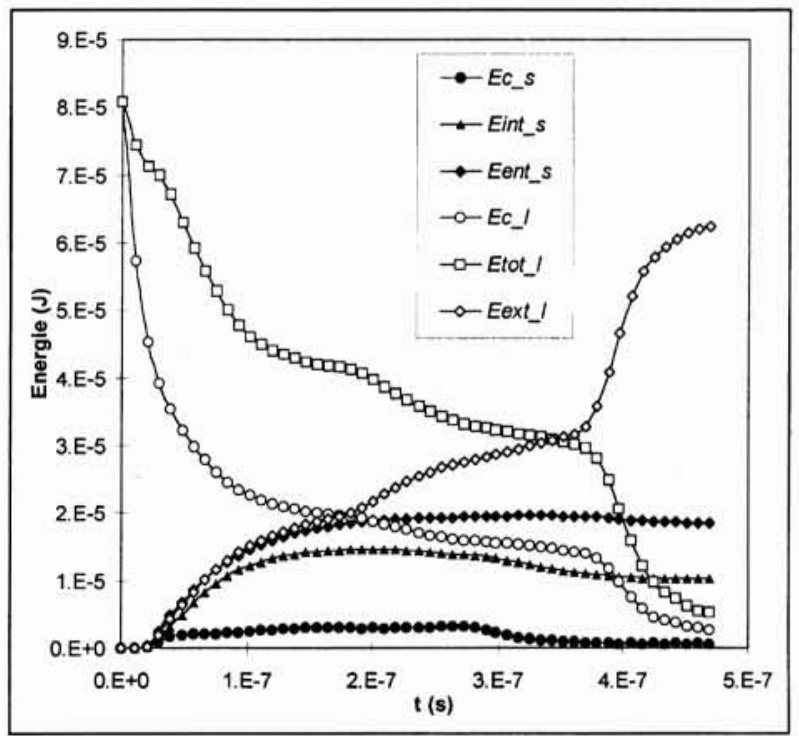

5. Evolution temporelle des différentes formes d'énergie intégrées sur les volumes liquide et solide. 
que plus de la moitié de l'énergie de l'onde se disperse directement dans le liquide dans la direction opposée à la surface solide et qu'une petite proportion supplémentaire est réfléchie par cette dernière. Pour cette amplitude d'impact, l'énergie des efforts intérieurs résiduelle représente environ la moitié du flux fourni au solide.

Une comparaison peut être faite avec l'énergie potentielle d'une bulle de rayon initial $R o$, donnée par l'approximation classique : $E b=4 / 3 \pi R o^{3}(P \infty-P v)$. On constate alors que pour une bulle de rayon initial de l'ordre de la distance à la paroi $(R o=0,07 \mathrm{~mm})$, le déséquilibre initial de pression $P \infty-P v$ devrait dépasser $40 \mathrm{MPa}$ pour atteindre le niveau d'énergie proche de $0,07 \mathrm{~mJ}$ mis en jeu lors de la création de l'empreinte simulée ci-dessus. Une telle valeur implique la mise en œuvre d'un processus complexe dans lequel une bulle de petite taille, proche de la paroi, implose sous la contrainte du champ de pression élevé émis par l'implosion de bulles voisines ou l'impact d'un microjet. A l'inverse, si l'on considère un déséquilibre initial de pression de $0,1 \mathrm{MPa}$, le rayon équivalent de la bulle de même énergie potentielle est de plus de $1 \mathrm{~mm}$, ce qui conduit à un rapport $\gamma=L R o$ de moins de 0,1 et une bulle quasiment hémisphérique. On retrouve ainsi les deux configurations érosives les plus probables proposées par Van der Meulen et Van Rennesse [14]

La même démarche peut être suivie pour l'analyse de la création d'une empreinte dans un échantillon de cuivre pur placé dans une cuve pressurisée et soumis à l'implosion d'une bulle générée par un éclateur [15]. Le cas retenu concerne une empreinte de $5 \mu \mathrm{m}$ de profondeur et $0,5 \mathrm{~mm}$ de rayon $R 10 \%$, créée lors de l'implosion sous une pression de 1,5 bars d'une bulle de rayon maximal $R o=3,6 \mathrm{~mm}$ générée à $4 \mathrm{~mm}$ de la paroi. L'énergie théorique disponible vaut alors $E b=29 \mathrm{~mJ}$. La simulation de l'empreinte a été réalisée par ajustements successifs de la position et de l'amplitude de l'onde de surpression dans l'eau et une concordance satisfaisante, tant en volume déformé qu'en profondeur a été obtenue pour une distance $L$ de $0,4 \mathrm{~mm}$ et une pression maximale en paroi de $1,9 \mathrm{GPa}$. On constate alors (fig. 6) que la géométrie de l'empreinte n'est pas parfaitement ajustée à celle relevée expérimentalement, en étant notamment un peu trop étroite dans sa partie supérieure. L'énergie totale de l'onde simulée est voisine de $16 \mathrm{~mJ}$, soit $2 / 3$ de l'énergie potentielle théorique initiale, ce qui semble extrêmement élevé. L'énergie échangée avec le matériau atteint la valeur de $3,2 \mathrm{~mJ}$, alors que celle subsistant sous forme permanente après la déformation plastique vaut $1,7 \mathrm{~mJ}$. Des simulations simplifiées antérieures [6] ont montré que l'énergie nécessaire à la création d'une empreinte est une fonction rapidement décroissante du temps de passage de l'onde de surpression lorsque celui-ci est petit. De même, des géométries d'empreintes plus évasées dans leur partie

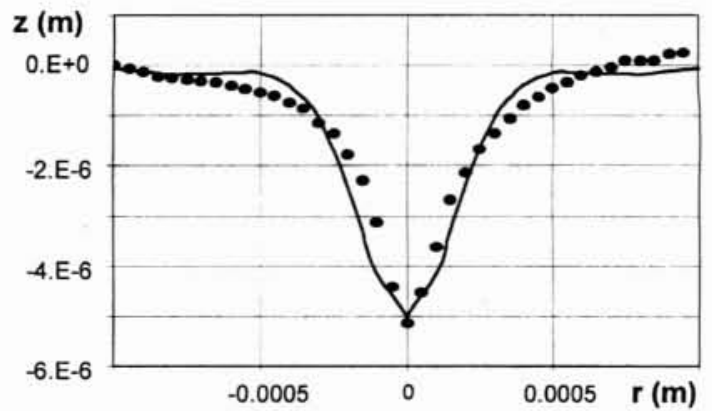

6. Géométrie d'empreinte simulée (trait) et relevée expérimentalement (points) sur un échantillon de cuivre.

supérieure, donc plus conformes à celles fournies par les relevés expérimentaux, ont été obtenues pour des temps de passage plus longs. Ces résultats tendent à montrer que le modèle FLUIDE actuel conduit vraisemblablement à une sous estimation de ce temps de passage, indiquant la nécessité d'améliorer la description physique du phénomène d'émission de l'onde.

\subsection{Influence des caractéristiques de l'onde}

Pour une même distance $L$, il est possible de générer des impacts d'amplitude variable. Ceux-ci conduisent à des empreintes de profondeurs différentes alors que le rayon $R 10 \%$ reste pratiquement inchangé. Pour une variation de $P$ max entre 0,37 et $5,8 \mathrm{GPa}$, la profondeur $h$ des empreintes obtenues est comprise entre 0,25 et $45 \mu \mathrm{m}$, leur rayon $R 10 \%$ restant de l'ordre de $L$ soit $70 \mu \mathrm{m}$. En fonction de la pression maximale $P \max$, on a tracé (fig. 7) d'une part l'évolution des différentes formes d'énergie et d'autre part celle de la proportion d'énergie échangée entre le liquide et le matériau, sous forme transitoire ou permanente. La proportion d'énergie fournie au matériau augmente avec l'amplitude de l'onde du fait du recul plus important de la paroi solide. De même, l'augmentation du niveau de plastification se traduit par la croissance du pourcentage d'énergie subsistant sous forme permanente. Pour la valeur de $P \max$ la plus élevée, près de la moitié de l'énergie de l'onde est transmise du fluide au matériau, au sein duquel la part dispersée par les ondes élastiques représente moins de $40 \%$, soit un « rendement » global pour la création de l'empreinte voisin de $30 \%$. Cette valeur n'atteint que $10 \%$ dans le cas d'une amplitude plus faible, avec Pmax de l'ordre de $1 \mathrm{GPa}$.
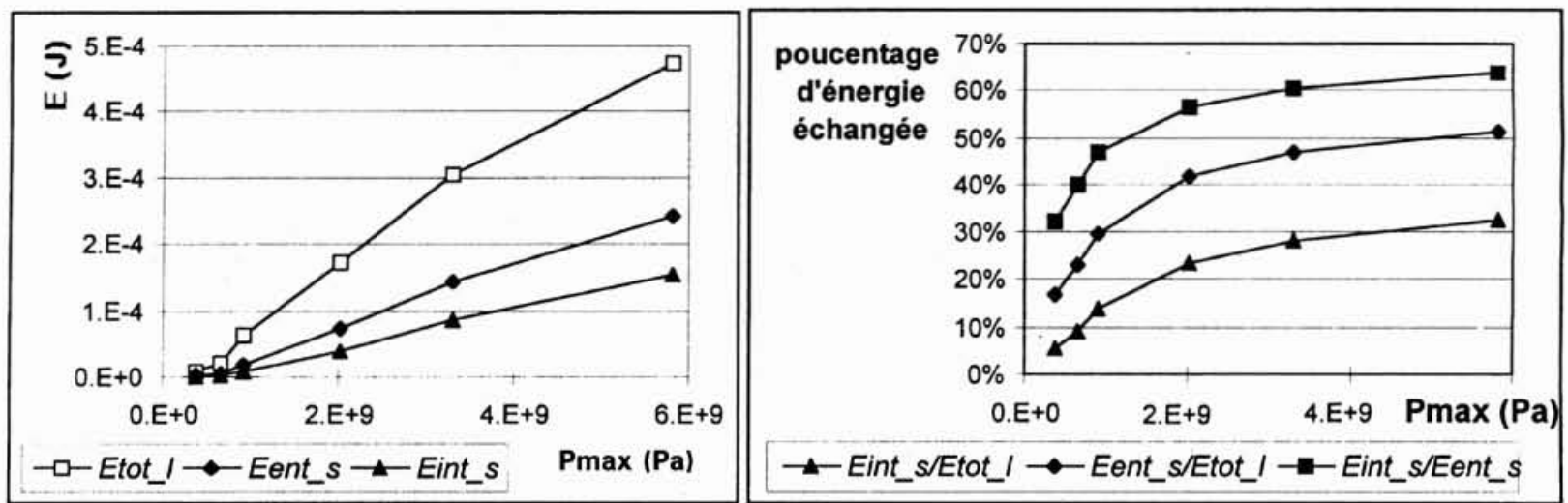

7. Evolution de différentes formes d'énergie et de leur proportion avec la pression maximale en paroi. 
L'influence de la valeur de $L$ est plus délicate à étudier dans la mesure où pour les mêmes conditions de génération de l'onde dans le liquide, les variations de la distance du centre d'émission à la paroi se traduisent par des modifications de la pression Pmax ressentie par celle-ci. Les fortes non linéarités du phénomène de propagation obligent à ajuster la vitesse initiale par tâtonnement pour reproduire des niveaux de pression Pmax équivalents d'un cas à un autre. Dans ces conditions on peut vérifier que l'effet de volume est prépondérant, avec les différentes formes d'énergie croissant environ comme $L^{3}$, le rayon $R 10 \%$ et la profondeur $h$ étant proportionnels à $L$. Lors de l'étude antérieure basée sur un modèle analytique simplifié de propagation d'une onde sphérique dans le liquide [6], l'analyse des empreintes simulées pour une large gamme des différents paramètres a montré qu'un facteur de similitude important était une expression adimensionnelle du temps de passage $\delta t$ de l'onde: Cliq $\delta t / L$, où $C l i q$ indique la vitesse de propagation. Le numérateur de cette expression n'étant plus un paramètre ajustable de notre calcul actuel, son influence n'a pas pu être confirmée ici.

\section{IV $\square$ CONCLUSION}

La modélisation numérique du couplage fluide-structure intervenant dans le phénomène d'érosion par cavitation représente un support pour l'analyse des échanges d'énergie conduisant à la génération d'une empreinte dans un solide. L'exploitation d'un tel modèle pour simuler l'interaction entre une onde de surpression sphérique et un matériau, permet en particulier d'estimer le « rendement énergétique » du processus. Pour l'ensemble des cas étudiés, celui-ci est compris entre 10 et $30 \%$, les plus fortes valeurs étant obtenues pour les amplitudes d'impact les plus grandes.

Avec le modèle d'onde de surpression sphérique étudié, la génération d'une empreinte nécessite l'émission de pics de pression dépassant largement le Gigapascal. L'énergie nécessaire ne semble alors pouvoir être fournie que par l'effondrement de bulles de grande taille, non sphériques, ou celui de bulles plus petites, mais soumises à un déséquilibre de pression de plusieurs Mégapascals. Une poursuite de ces travaux devra consister à améliorer la description des phénomènes d'émission d'ondes de surpression, pour prendre en compte de façon plus complète le comportement physique du fluide dans des conditions aussi extrêmes de vitesse et de pression.

\section{REMERCIEMENTS}

Le département Machines de la Direction des Etudes et Recherches d'Electricité de France (EDF) participe depuis plusieurs années aux recherches effectuées au laboratoire sur l'analyse et la modélisation de l'érosion de cavitation. Le développement du modèle SOLIDE a été réalisé en majeure partie dans le cadre d'une convention avec le Centre Technique des Industries Mécaniques (CETIM). Les améliorations récentes du code FLUIDE ont été effectuées en 1995 lors du stage de T. Defrance, élève ingénieur à l'Institut National Polytechnique de Grenoble.

\section{REFERENCES}

[1] KNAPP (1955), - « Recent investigations of cavitation and cavitation damage » Trans. ASME, Vol. 77.

[2] Haмmiт (1979). - «Cavitation erosion : the state of the art and predicting capability " Applied Mech. Review, Vol. 32-6.

[3] Lecoffre, Marcoz, Franc, Michel (1985), - « Tentative procedure for scaling cavitation damage " ASME Int. Symp. on Cavitation in Hydraulic Structure and Turbomachinery, Albuquerque.

[4] Franc, Michel, Karimi (1991). - «An analytical method for the prediction of cavitation erosion " Cavitation'91 Symposium, ASME-FED, Vol. 116.

[5] ReBoud (1987), - « Réponse impulsionnelle d'un milieu élastoplastique : application à l'étude de l'érosion de cavitation ", Thèse de Doctorat (Mécanique), INPG, Grenoble.

[6] Fortes Patella (1994). - « Analyse de l'érosion de cavitation par simulation numérique d'impacts ", Thèse de Doctorat (Mécanique), INPG, Grenoble.

[7] Reboud, Fortes Patella, Dorey (1991). - «Simulation of cavitation impact damage on an elastoplastic solid " Cavitation and Multiphase Flow Forum, ASME-FED, Vol. 109.

[8] Guelin (1980). - « Remarques sur l'hystéresis mécanique » Journal de mécanique, Vol. 19-2.

[9] ReBoud, Guelin (1988). - « Impact response of an elastoplastic medium », Mech Research Comm., Vol. 15(4).

[10] CheVRILler (1990). - « Simulation numérique de l'interaction arc électrique - écoulement gazeux dans les disjoncteurs moyenne et haute tension " Thèse de Doctorat (Mathématiques appliquées), INPG, Grenoble.

[11] Thompson (1987 et 1990). — « Time dependent boundary conditions for hyperbolic systems ", J. of Computational Physics., Vol. 68 (partie I) et Vol. 89 (partie II).

[12] Vogel, Lauterborn, Timm (1989). — «Optical and acoustic investigation of the dynamics of laser-produced cavitation bubbles near a solid boundary ", Journal of Fluid Mechanics, Vol. 206

[13] WARD, Emmony (1990), - «The energies and pressures of acoustic transients associated with optical cavitation in water » Journal of Modern Optics, Vol 257

[14] Van Der Meulen, Van Remmesse (1993). - « The collapse of laser-indiced bubbles near a solid boundary and the generation of pressure pulses ", Cavitation and Multiphase Flow Forum, ASME-FED, Vol. 153.

[15] DOREY, NiEnAltowsKa (1988). — « Empreintes dues à l'implosion de bulles isolées : étude paramétrique expérimentale ", la Houille Blanche, Vol. 7/8, 1988.

[16] Fortes Patella, Reboud (1995), - « A new approach to evaluate the cavitation erosion power ", Proceedings of the Cav'95 Symposium, Deauville, Mai 1995. 